\title{
$k$-INVARIANTS FOR K-THEORY OF CURVES OVER GLOBAL FIELDS
}

\author{
D. Arlettaz, G. Banaszak
}

\section{Introduction.}

Let $X$ be a connected simple CW-complex and let $\alpha_{n}: X \rightarrow X[n]$ denote the $n$-th Postnikov section of $X$ for any positive integer $n: X[n]$ is the CW-complex obtained from $X$ by killing the homotopy groups of $X$ in dimensions $>n$, more precisely by adjoining cells of dimensions $\geq n+2$ such that $\pi_{j}(X[n])=0$ for $j>n$ and $\left(\alpha_{n}\right)_{*}: \pi_{j}(X) \rightarrow \pi_{j}(X[n])$ is an isomorphism for $j \leq n$. The Postnikov $k$-invariants of $X$ are cohomology classes $k^{n+1}(X) \in H^{n+1}\left(X[n-1] ; \pi_{n}(X)\right)$, for $n \geq 2$, which provide the necessary information for the reconstruction of $X$, up to a weak homotopy equivalence, from its homotopy groups (see for instance [W], Section IX.2 for a definition).

In this paper we extend our results of $[\mathrm{AB}]$ concerning $k$-invariants to the case of algebraic curves over global fields. In section 2 we state general results about $k$ invariants and we discuss the cases when they are of finite order. Then in section 3 we consider the case of $k$-invariants of Quillen and Étale K-theory spaces and we explain why they are of finite order. In section 4 we prove our main result:

Theorem 4.9. Let $X$ be a smooth, proper and geometrically irreducible curve of genus $g$, defined over a global field $F$ and $\mathcal{X}$ be a regular and proper model of $X$ over $O_{F, S_{l}}$. Let $\mathcal{X}_{v}$ be the special fibre of $\mathcal{X}$ at a prime $v$. Then for $n \geq 1$ and $l \gg 0$ the order of $k_{\text {et }}^{2 n+1}(\mathcal{X})$ divides the order of $k_{\text {et }}^{2 n+1}\left(\mathcal{X}_{v}\right)$ for infinitely many $v \notin S_{l}$.

To prove Theorem 4.9 we need a technical result about Galois cohomology of a compatible family of $l$-adic representations (see Lemma 4.1 and Remark 4.2). From this we conclude that for $l \gg 0$ the $\mathbb{Z}_{l}$ module $K_{2 n}^{e t}(\mathcal{X})$ has no $l$-torsion (see Lemma 4.3). Note that Lemma 4.3 gives some evidence that the conjecture of QuillenLichtenbaum for $K_{2 n}^{e t}(\mathcal{X})$ (see Remark 4.5) and the conjecture about finite generation of $K_{2 n}^{e t}(\mathcal{X})$ might be true. Other important technical ingredients in the proof of Th. 4.9 are: the compatibility of $k$-invariants (cf. eg. diagrams 2.4 and 4.11 ) and good control of the reduction map for étale K-theory [BGK3, Th. 3.1]. Theorem 4.9 has the following corollary in which we numerically estimate the orders of $k$-invariants. 
Corollary 4.19. Let $X$ be a smooth, proper and geometrically irreducible curve of genus $g$, defined over a global field $F$ and $\mathcal{X}$ be a regular and proper model of $X$ over $O_{F, S_{l}}$. For every $n \geq 1$ and $l \gg 0$ the order of $k_{e t}^{2 n+1}(\mathcal{X})$ divides the number $\left|P_{v}\left(q_{v}^{-n}\right)\right|_{l}^{-1}$ for infinitely many $v \notin S_{l}$, where $P_{v}(t)=\operatorname{det}\left(t I d_{T_{l}\left(J_{X}\right)}-F r_{v}\right)$ is the characteristic polynomial of the Frobenius $F r_{v}$ acting on the Tate module of the Jacobian of $X$. If the Quillen-Lichtenbaum conjecture holds for $\mathcal{X}$ and $\mathcal{X}_{v}$ for all $v \notin S_{l}$, then for every $n \geq 1$ and $l \gg 0$ the l-part of the order of $k^{2 n+1}(\mathcal{X})$ divides the number $\left|P_{v}\left(q_{v}^{-n}\right)\right|_{l}^{-1}$ for infinitely many $v \notin S_{l}$.

It is explained in Remark 4.2 that the assumption $l \gg 0$ can be made numerically explicit. We finish the paper with discussion of $k$-invariants associated with odd K-groups and we get similar results to Th. 4.9 and Corollary 4.19 (cf. Th. 4.25 and Corollary 4.26).

\section{Basic facts about $k$-invariants.}

The main property of $k$-invariants is that $X[n]$ is the homotopy fiber of the map $X[n-1] \rightarrow K\left(\pi_{n}(X), n+1\right)$ corresponding to the cohomology class $k^{n+1}(X) \in$ $H^{n+1}\left(X\left[n-1 \mid ; \pi_{n}(X)\right)\right.$, for $n \geq 2$. In other words, there is a commutative diagram of fibrations

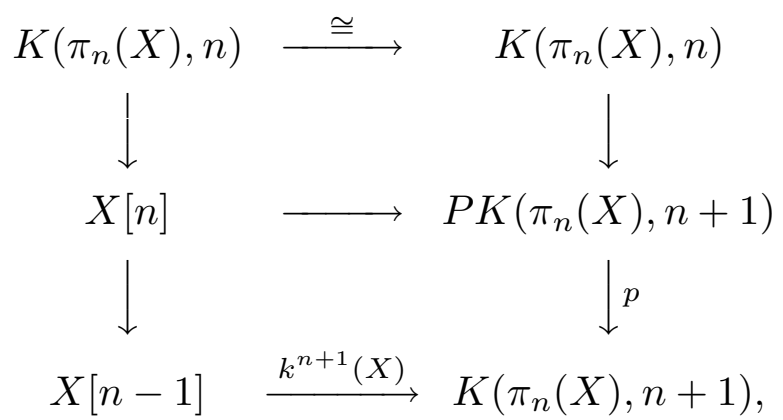

in which the right column is the path fibration over the Eilenberg-MacLane space $K\left(\pi_{n}(X), n+1\right)$ and the bottom square is a homotopy pull-back. Consequently, the knowledge of $X[n-1], \pi_{n}(X)$ and $k^{n+1}(X)$ enables us to construct the next homotopical approximation $X[n]$ of $X$.

It is then obvious that the vanishing of $k^{n+1}(X)$ implies that $X[n] \cong X[n-1] \times$ $K\left(\pi_{n}(X), n\right)$ and that the Hurewicz homomorphism $h_{n}: \pi_{n}(X) \rightarrow H_{n}(X ; \mathbb{Z})$ is split injective (see $[\mathrm{A}]$, Lemma 7.3). It is of particular interest to get information on the order of the $k$-invariant $k^{n+1}(X)$ in the group $H^{n+1}\left(X[n-1] ; \pi_{n}(X)\right)$, because the previous splitting result can be generalized as follows for nonvanishing $k$-invariants which are of finite order (see [A], Proposition 7.10).

Theorem 2.1. Let $X$ be a connected simple $C W$-complex, $n$ an integer $\geq 2$ and $\rho$ a positive integer. The following assertions are equivalent:

(1) $\rho k^{n+1}(X)=0$ in $H^{n+1}\left(X[n-1] ; \pi_{n}(X)\right)$.

(2) There is a map

$$
f_{n}: X \rightarrow K\left(\pi_{n}(X), n\right)
$$


such that the induced homomorphism $\left(f_{n}\right)_{*}: \pi_{n}(X) \rightarrow \pi_{n}(X)$ is multiplication by $\rho$.

(3) There is a homomorphism $\theta_{n}: H_{n}(X ; \mathbb{Z}) \rightarrow \pi_{n}(X)$ such that the composition

$$
\pi_{n}(X) \stackrel{h_{n}}{\longrightarrow} H_{n}(X ; \mathbb{Z}) \stackrel{\theta_{n}}{\longrightarrow} \pi_{n}(X)
$$

where $h_{n}$ denotes the Hurewicz homomorphism, is multiplication by $\rho$.

Lemma 2.2. If $X$ is an infinite loop space then $k^{n+1}(X)$ is of finite order for $n \geq 2$.

Proof. It follows by Theorem 7.15 of $[\mathrm{A}]$.

Theorem 2.3. Let

$$
F \rightarrow E \rightarrow B
$$

be a fibration. Assume that both $\pi_{n-1}(F)$ and $\pi_{n}(F)$ are torsion groups and that $k^{n+1}(B)$ has finite order. Then $k^{n+1}(E)$ has finite order.

Proof. Consider the exact sequence

$$
\pi_{n}(F) \stackrel{i_{*}}{\longrightarrow} \pi_{n}(E) \stackrel{\pi_{*}}{\longrightarrow} \pi_{n}(B) \stackrel{\partial}{\longrightarrow} \pi_{n-1}(F)
$$

Define the groups $I_{1}:=\operatorname{Im}\left(i_{*}\right), I_{2}:=\operatorname{Im}\left(\pi_{*}\right), I_{3}:=\operatorname{Im}(\partial)$. Observe that $I_{1}$ and $I_{3}$ are torsion groups. In addition we get two short exact sequences

$$
\begin{aligned}
& 0 \rightarrow I_{1} \rightarrow \pi_{n}(E) \rightarrow I_{2} \rightarrow 0, \\
& 0 \rightarrow I_{2} \rightarrow \pi_{n}(B) \rightarrow I_{3} \rightarrow 0 .
\end{aligned}
$$

These exact sequences yield exact sequences in cohomology

$$
\begin{gathered}
H^{n+1}\left(E[n-1], I_{1}\right) \rightarrow H^{n+1}\left(E[n-1], \pi_{n}(E)\right) \rightarrow H^{n+1}\left(E[n-1], I_{2}\right), \\
H^{n}\left(E[n-1], I_{3}\right) \rightarrow H^{n+1}\left(E[n-1], I_{2}\right) \rightarrow H^{n+1}\left(E[n-1], \pi_{n}(B)\right) .
\end{gathered}
$$

Because $I_{1}$ and $I_{3}$ are torsion groups, it is clear from the above sequences that for $k^{n+1}(E)$ to have finite order it is enough to check that its image via the composition of the right maps in the exact sequences has finite order in $H^{n+1}\left(E[n-1], \pi_{n}(B)\right)$. Now the Theorem follows by applying the following diagram:

$$
\begin{gathered}
H^{n+1}\left(B[n-1], \pi_{n}(B)\right) \\
\downarrow \alpha \\
H^{n+1}\left(E[n-1], \pi_{n}(B)\right) \\
\uparrow \beta \\
H^{n+1}\left(E[n-1], \pi_{n}(E)\right)
\end{gathered}
$$

and the assumption that $k^{n+1}(B)$ has finite order because $\alpha\left(k^{n+1}(B)\right)=\beta\left(k^{n+1}(E)\right)$ by [W] p. 424 . 
Example 2.5. We observe that if all $k$-invariants of $F$ and all $k$-invariants of $B$ are of finite order it does not imply that all $k$-invariants of $E$ are of finite order. Namely we can take $F$ and $B$ to be Eilenberg-McLane spaces then the only $k$ invariant of $E$ can be of infinite order.

\section{Finite order of $k$-invariants for K-theory of schemes.}

Let $\mathcal{E}$ be an exact category. Quillen [Q] constructed the space $B Q \mathcal{E}$ and defined K-theory of $\mathcal{E}$ as follows: $K_{n}(\mathcal{E}):=\pi_{n}(B Q \mathcal{E})$. We will denote the k-invariant for the space $B Q \mathcal{E}$ as follows: $k^{n+1}(\mathcal{E}):=k^{n+1}(B Q \mathcal{E})$.

Lemma 3.1. If $\mathcal{E}$ is an exact category then $B Q \mathcal{E}$ is an infinite loop space.

Proof. It follows by application of Waldhausen's construction (see e.g. [We], chapter IV, Remark 4.5.1).

In this way by Lemma 2.2 the k-invariats $k^{n}(\mathcal{E})$ are of finite order for any $n \geq 3$ and any exact category $\mathcal{E}$. Let $X$ be a scheme and let $\mathbb{M}(X)$ be the category of finitely generated coherent sheaves on $X$ and let $\mathbb{P}(X)$ be the category of finitely generated, localy free $\mathcal{O}_{X}$ modules on $X$. Both $\mathbb{P}(X)$ and $\mathbb{M}(X)$ are exact categories and Quillen [Q] defined $K^{\prime}$-theory and $K$-theory of a scheme $X$ as follows: $K_{n}^{\prime}(X):=$ $\pi_{n}(B Q \mathbb{M}(X))$ and $K_{n}(X):=\pi_{n}(B Q \mathbb{P}(X))$. When $X$ is a regular scheme Quillen [Q] proved that $K_{n}^{\prime}(X)=K_{n}(X)$. We will simplify the notation for $k$-invariants of $\mathrm{K}$-theory spaces of schemes in the following way: $k^{\prime, n}(X):=k^{n}(B Q \mathbb{M}(X))$ and $k^{n}(X):=k^{n}(B Q \mathbb{P}(X))$. By lemma 2.2 the $k$-invariants $k^{n}(X)$ and $k^{\prime}, n(X)$ have finite order for any $n \geq 3$. If $X=\operatorname{spec} R$, where $R$ is a unital, commutative ring, then we will write $k^{n}(R):=k^{n}(X)$. For a prime $l$ let $\mathcal{K}^{e t}(X)$ denote the Dwyer and Friedlander étale K-theory space of a scheme $X / \mathbb{Z}[1 / l]$. Let $K_{n}^{e t}(X)$ denote the étale K-theory group of a scheme $X$ over spec $\mathbb{Z}\left[\frac{1}{l}\right]$ cf. $[\mathrm{DF}]$. Let $k_{e t}^{n}(X):=k^{n}\left(\mathcal{K}^{e t}(X)\right)$. By Proposition $4.5[\mathrm{DF}] \mathcal{K}^{e t}(X)$ is homotopy equivalent to an $r-t h$ loop space for any $r$ so for any $n \geq 3$ the $\mathrm{k}$-invariant $k_{e t}^{n}(X)$ is of finite order for any scheme $X / \mathbb{Z}[1 / l]$. Using a technique from $[\mathrm{AB}]$ we can prove the following basic properties of $k$-invariants of schemes.

Proposition 3.2. Let $X / S$ be a scheme over a scheme $S$. Assume that $X$ has a point over $S$. Then for $n \geq 2$ the order of $k^{n+1}(X)\left(k_{e t}^{n+1}(X)\right.$ resp.) is divisible by the order of $k^{n+1}(S)\left(k_{e t}^{n+1}(S)\right.$ resp. $)$.

Proof. We do the proof for algebraic K-theory. The étale K-theory case is treated analogously. Since the composition of maps $S \rightarrow X \rightarrow S$ is the identity by assumption, it follows that the composition

$$
B Q \mathbb{P}(S)[n-1] \rightarrow B Q \mathbb{P}(X)[n-1] \rightarrow B Q \mathbb{P}(S)[n-1]
$$


is also the identity. Hence the map $\alpha$ in the diagram below is split injective

$$
\begin{gathered}
H^{n+1}\left(B Q \mathbb{P}(S)[n-1], K_{n}(S)\right) \\
\downarrow \alpha \\
H^{n+1}\left(B Q \mathbb{P}(X)[n-1], K_{n}(S)\right) \\
\uparrow_{\beta} \\
H^{n+1}\left(B Q \mathbb{P}(X)[n-1], K_{n}(X)\right)
\end{gathered}
$$

Corollary 3.3. Let $X / \mathbb{F}$ be a scheme over a finite field $\mathbb{F}$. Assume that $X$ has a point over $\mathbb{F}$. Then for $n \geq 2$ the order of $k^{2 n}(X)$ is divisible by the order of $k^{2 n}(\mathbb{F})$ which is equal to $\operatorname{gcd}((n-1) !,|\mathbb{F}|-1)$. Also the order of $k_{\text {et }}^{2 n}(X)$ is divisible by the order of $k_{e t}^{2 n}(\mathbb{F})$

Proof. The proof follows from Lemma 2.2, Lemma 3.2 and Theorem C of $[\mathrm{H}]$.

\section{4. $k$-invariants for K-theory of curves over global fields.}

Let us start with some remarks on Galois cohomology and $l$-adic representations.

Let $F$ be a global field and consider for each $l$ a free, finitely generated $\mathbb{Z}_{l}$ module $T_{l}$ and an $l$ adic representation $\rho_{l}: G_{F} \rightarrow G L\left(T_{l}\right)$. For each $l$ let $S_{l}$ be a finite set of valuations of $F$ containing all primes over $l$ in the number field case. Let $V_{l}:=T_{l} \otimes_{\mathbb{Z}_{l}} \mathbb{Q}_{l}$ and $A_{l}:=V_{l} / T_{l}$. The representation $\rho_{l}$ induces the following $l$-adic represenatation: $\rho_{l}^{0}: G_{F} \rightarrow G L\left(V_{l}\right)$. Representation $\rho_{l}^{0}$ is called rational (cf. [Se]) if $\rho_{l}^{0}$ is unramified outside $S_{l}$ and for each finite valuation $v \notin S_{l}$ the characteristic polynomial

$$
P_{v, \rho_{l}}(t)=\operatorname{det}\left(t \operatorname{Id}_{V_{l}}-F r_{v}\right)=\operatorname{det}\left(t \operatorname{Id}_{T_{l}}-F r_{v}\right)
$$

of the Frobenius at $v$ has coefficients in $\mathbb{Q}$. Since $\rho_{l}^{0}$ is unramified at a given prime $v$ iff $\rho_{l}$ is unramified at this prime, we will also say that $\rho_{l}$ is rational whenever $\rho_{l}^{0}$ is rational. The family of $l$-adic representations $\left(\rho_{l}^{0}\right)_{l}$ is called strictly compatible [Se] I, 2.3 if there exists a finite set of places $S$ of $F$ such that each represenatation $\rho_{l}^{0}$ is rational with $S_{l}=S \cup\{$ primes over $l\}$ in number field case and $S_{l}=S$ in function field case and for any primes $l$ and $l^{\prime}$ and $v \notin S_{l} \cup S_{l^{\prime}}$ there is an equality $P_{v, \rho_{l}}(t)=P_{v, \rho_{l^{\prime}}}(t)$. For strictly compatible family of representations the polynomial $P_{v, \rho_{l}}(t)$ is independant of prime number $l$ hence for each $v \notin S$ we can put:

$$
P_{v}(t):=P_{v, \rho_{l}}(t)
$$

for any $l$ such that $v \notin S_{l}$. Again we easily observe that it is justified to say that the family $\left(\rho_{l}\right)_{l}$ is strictly compatible instead of saying that $\left(\rho_{l}^{0}\right)_{l}$ is strictly compatible. 
Lemma 4.1. Assume that the family of representations $\left(\rho_{l}\right)_{l}$ is strictly compatible and all roots of $P_{v}(t)$ in $\overline{\mathbb{Q}}$ are different from 1 . Then $H^{1}\left(G_{F, S_{l}} ; T_{l}\right)_{l-\text { tor }}=0$ for almost all $l$.

Proof. Let $\overline{\mathbb{Z}}_{l}$ be the integers in $\overline{\mathbb{Q}}_{l}$. We can write

$$
P_{v}(t)=\left(t-\alpha_{1}\right)\left(t-\alpha_{2}\right) \ldots\left(t-\alpha_{r}\right) \in \overline{\mathbb{Q}}[t] .
$$

Put $L:=\mathbb{Q}\left(\alpha_{1}, \alpha_{2}, \ldots, \alpha_{r}\right) . L$ is the splitting field of $P_{v}(t)$. Choose $l \gg 0$ such that $\alpha_{i}$ are integers over $l$ and $\alpha_{i}-1$ are units over $l$ for all $1 \leq i \leq r$. Let $\bar{F} r_{v}: A_{l}[l] \rightarrow A_{l}[l]$ be the automorphism of $\mathbb{F}_{l}$ vector space $A_{l}[l]$ induced by the automorphism $F r_{v}: T_{l} \rightarrow T_{l}$ of free $\mathbb{Z}_{l}$ module $T_{l}$. Let $\wp$ be a prime in $O_{L}$ over $l$. Put $k_{\wp}:=O_{L} / \wp$. Consider the automorphism

$$
\bar{F} r_{v} \otimes 1: A_{l}[l] \otimes_{\mathbb{F}_{l}} k_{\wp} \rightarrow A_{l}[l] \otimes_{\mathbb{F}_{l}} k_{\wp}
$$

of $k_{\wp}$ vector space $A_{l}[l] \otimes_{\mathbb{F}_{l}} k_{\wp}$. Observe that by definition of the splitting field $L$ we have:

$$
\bar{P}_{v}(t)=\operatorname{det}\left(t \operatorname{Id}_{A_{l}[l] \otimes_{\mathbb{F}_{l}} k_{\wp}}-\bar{F} r_{v} \otimes 1\right)=\left(t-\bar{\alpha}_{1}\right)\left(t-\bar{\alpha}_{2}\right) \ldots\left(t-\bar{\alpha}_{r}\right) \in k_{\wp}[t]
$$

where $\bar{P}_{v}(t)$ is the polynomial obtained from $P_{v}(t)$ by reducing its coefficients modulo $l$ and $\bar{\alpha}_{i}$ are the images of $\alpha_{i}$ in $k_{\wp}$. We observe that the automorphism $\bar{F} r_{v}$ cannot have a fix point because it would imply that the automorphism $\bar{F} r_{v} \otimes 1$ has a fix point which is equivalent to the statement that the polynomial $\bar{P}_{v}(t)$ has $1 \in k_{\wp}$ as a root. This means that $\bar{\alpha}_{i}=1$ for some $1 \leq i \leq r$. This would imply that $\alpha_{i}-1$ is not a unit over $l$, a contradiction. Hence $\bar{F} r_{v}$ cannot have a fix point which imply that $A_{l}[l]^{G_{F, S_{l}}}=0$ for $l \gg 0$ since for $v \notin S_{l}$ we can consider $F r_{v}$ as an element of $G_{F, S_{l}}$. So applying cohomology to the exact sequence

$$
0 \rightarrow T_{l} \rightarrow V_{l} \rightarrow A_{l} \rightarrow 0
$$

we get the exact sequence:

$$
0 \rightarrow A_{l}^{G_{F, S_{l}}} \rightarrow H^{1}\left(G_{F, S_{l}} ; T_{l}\right) \rightarrow H^{1}\left(G_{F, S_{l}} ; V_{l}\right)
$$

cf. [BGK1], which shows that $H^{1}\left(G_{F, S_{l}} ; T_{l}\right)_{l-t o r}=A_{l}^{G_{F, S_{l}}}=0$.

Remark 4.2. The statement that $l \gg 0$ in Lemma 4.1 can be made much more explicit. It is clear from the proof of Lemma 4.1. Simply we can pick any place $v_{0} \notin S$. In the number field case we take $l_{0}$ to be the prime in $\mathbb{Z}$ below $v_{0}$. Then we do the proof with the eigenvalues of $F r_{v_{0}}$ which are the roots $\alpha_{1}^{0}, \ldots, \alpha_{r}^{0}$ of the polynomial $P_{v_{0}}(t)$.

In the number field case the assumption $l \gg 0$ means that $l$ is not below any prime in $S_{l_{0}}$, and $\alpha_{i}^{0}-1 \in \overline{\mathbb{Z}}_{l}^{\times}, \alpha_{i}^{0} \in \overline{\mathbb{Z}}_{l}$ for each $1 \leq i \leq r$. 
In the function field case the assumption $l \gg 0$ means that $l \neq$ char $F$ and $\alpha_{i}^{0}-1 \in$ $\overline{\mathbb{Z}}_{l}^{\times}, \alpha_{i}^{0} \in \overline{\mathbb{Z}}_{l}$ for each $1 \leq i \leq r$.

In all results below the assumption $l \gg 0$ can be made explicit in the same way as we did it above for the case of Lemma 4.1.

Now consider $X$ a smooth, proper and geometrically irreducible algebraic variety defined over a global field $F$. Let $l$ be an odd rational prime different from the characteristic of the field $F$. Let $S$ be the set of places of $F$ which consists of places of bad reduction of $X$ and places at infinity. Denote by $\mathcal{X}$ a regular and proper model of $X$ over the ring $O_{F, S}$ of $S$-integers of $F$. Let $S_{l}=S$ in function field case and $S_{l}=S \cup\{$ places lying over $l\}$ in the number field case. For a prime $v$ of $O_{F, S_{l}}$ let $F_{v}$ denote the completion of $F$ at $v k_{v}:=O_{F, S_{l}} / v$. Let $\bar{F}$ (resp. $\bar{k}_{v}$ ) denote algebraic closure of $F$ (resp. $k_{v}$ ). Let $\mathcal{X}_{v}$ be the fiber of $\mathcal{X}$ over a prime $v$ of $O_{F, S_{l}}$. By [Mi], VI, Cor. 4.2 there are natural isomorphisms of $\mathbb{Z}_{l}\left[G\left(\bar{F}_{v} / F_{v}\right)\right]$-modules:

$$
H^{i}\left(\bar{X} ; \mathbb{Z}_{l}(j)\right) \cong H^{i}\left(\overline{\mathcal{X}}_{v} ; \mathbb{Z}_{l}(j)\right)
$$

for all $i \geq 0$ and all $j \in \mathbb{Z}$, where $\bar{X}=X \otimes_{F} \bar{F}$ and $\overline{\mathcal{X}}_{v}=\overline{\mathcal{X}}_{v} \otimes_{k_{v}} \bar{k}_{v}$. Since the innertia subgroup $I_{v} \subset G\left(\bar{F}_{v} / F_{v}\right)$ acts trivially on the group on the right side of the above isomorphisms, it shows that the action of $G_{F}$ on the group on the left factors through $G_{F, S_{l}}$. Taking $T_{l}:=H^{i}\left(\bar{X} ; \mathbb{Z}_{l}(j)\right) / l-t o r$ we get a family of $l$-adic representations. This family is strictly compatible because of the proof of the Weil conjecture by Deligne $[\mathrm{D}]$ and the descussion above. It was conjectured by Jannsen $[\mathrm{J}]$, Conjecture 1, p. 317 that $H^{2}\left(G_{F, S_{l}} ; H^{i}\left(\bar{X} ; \mathbb{Z}_{l}(j)\right)\right)$ is finite for $i+1<j$ or $i+1>2 j$. Jannsen verified this conjecture in function field case $[\mathrm{J}]$, Theorem 1, p. 335. Now consider the special case when $X$ is a smooth, proper and geometrically irreducible curve of genus $g$, defined over a global field $F$. Let $J_{X}$ denote the Jacobian variety of $X$ and let $T_{l}\left(J_{X}\right)$ denote the Tate module of the Jacobian. Then one checks that there is a natural isomorphism of $\mathbb{Z}_{l}\left[G_{F, S_{l}}\right]$ modules:

$$
H^{1}\left(\bar{X} ; \mathbb{Z}_{l}(1)\right) \cong T_{l}\left(J_{X}\right) .
$$

Hence according to Jannsen [J], Conjecture 1, p. 317, we expect the group $H^{2}\left(G_{F, S_{l}} ; T_{l}\left(J_{X}\right)(n+1)\right)$ to be finite for all $n>0$. Hence if $F$ is a function field then by $[\mathrm{J}]$, Theorem 1 , p. 335 the group $H^{2}\left(G_{F, S_{l}} ; T_{l}\left(J_{X}\right)(n+1)\right)$ is finite for all $n>0$.

Lemma 4.3. Let $X$ be a smooth, proper and geometrically irreducible curve of genus $g$, defined over a global field $F$ and let $\mathcal{X}$ be a regular and proper model of $X$ over $O_{F, S_{l}}$. For $n>0$ and for $l \gg 0$ the $\mathbb{Z}_{l}$-modules $K_{2 n}^{\text {et }}(\mathcal{X})$ are finitely generated, torsion free.

Proof. Put $T_{l}:=T_{l}\left(J_{X}\right)(n)$. By the diagrams (3.1) and (3.2) of the proof of Theorem 3.1 in [BGK3] there is a natural, surjective map

$$
K_{2 n}^{e t}(\mathcal{X}) \rightarrow H^{1}\left(G_{F, S_{l}} ; T_{l}\right)
$$


Observe that in the number field case by Th. 4 [So1] and Th. 8.7 [DF] the $H^{2}\left(G_{F, S_{l}} ; \mathbb{Z}_{l}(n+1)\right)$ is zero for $l \gg 0$ In the function field case $H^{2}\left(G_{F, S_{l}} ; \mathbb{Z}_{l}(n+1)\right)$ is zero for $l \gg 0$ becasue of Th. 8.9 [DF], Prop. 3.2 [Q1], Cor. 1, p.330 [So2]. Hence by diagrams (3.1) and (3.2), of the proof of Theorem 3.1 in [BGK3], the map (4.4) is an isomorphism for $l \gg 0$. By theorem of Deligne [D], the characteristic polynomial of $F r_{v}$ acting on $V_{l}$ has rational coefficients and its eigenvalues in this case are algebraic numbers with absolute values $N(v)^{1 / 2-n}$. Hence the eigenvalues are different from 1 . In addition by [ST] (or the discussion before this lemma) and by [D] the family of $l$-adic representations for $T_{l}:=T_{l}\left(J_{X}\right)(n)$ is strictly compatible. So by lemma 4.1 we get $H^{1}\left(G_{F, S_{l}} ; T_{l}\right)_{l-t o r}=0$ for $l \gg 0$, hence the isomorphism (4.4) for $l \gg 0$ shows that $K_{2 n}^{e t}(\mathcal{X})_{l-t o r}=0$ for $l \gg 0$. Finite generation of the groups $K_{2 n}^{e t}(\mathcal{X})$ follows by [DF] Prop. 5.1, p. 260.

Remark 4.5. Let us return for a while to the general case when $X$ is a smooth, proper and geometrically irreducible algebraic variety defined over a global field $F$. Note that according to the Beilinson conjectures the Quillen $K$-group $K_{m}(\mathcal{X})$ is expected to be finitely generated. The Quillen-Lichtenbaum conjecture (cf. [FW] p. 57) predicts that the Dwyer-Friedlander map [DF]:

$$
K_{m}\left(X ; \mathbb{Z} / l^{k}\right) \rightarrow K_{m}^{e t}\left(X ; \mathbb{Z} / l^{k}\right)
$$

is an isomorphism for $m \geq 3$. Hence by the localization sequences in K-theory the conjectures imply that for $m \geq 3$ there is an isomorphism:

$$
K_{m}(\mathcal{X}) \otimes_{\mathbb{Z}} \mathbb{Z}_{l} \rightarrow K_{m}^{e t}(\mathcal{X})
$$

Observe that if (4.7) is an isomorphism for $l \gg 0$ and $K_{m}(\mathcal{X})$ is finitely generated then Lemma 4.3 follows. Our proof of Lemma 4.3 does not appeal to these conjectures. Let us mention that by [DFST] the map (4.6) is surjective for $m \gg 0$.

From now on $X$ is a smooth, proper and geometrically irreducible curve of genus $g$, defined over a global field $F$ and $\mathcal{X}$ is a regular and proper model of $X$ over $O_{F, S_{l}}$.

For places $v \notin S_{l}$, we consider the reduction map:

$$
r_{v}: K_{2 n}^{e t}(\mathcal{X}) \rightarrow K_{2 n}^{e t}\left(\mathcal{X}_{v}\right)
$$

induced on étale K-theory by the injection $\mathcal{X}_{v} \hookrightarrow \mathcal{X}$ of the special fiber at $v$.

Theorem 4.9. For $n \geq 1$ and $l \gg 0$ the order of $k_{e t}^{2 n+1}(\mathcal{X})$ divides the order of $k_{e t}^{2 n+1}\left(\mathcal{X}_{v}\right)$ for infinitely many $v$.

Proof. Consider the reduction map $r_{v}$ (4.8). Since $K_{2 n}^{e t}(\mathcal{X}) \cong \oplus_{i=1}^{r} \mathbb{Z}_{l}$ for some $r$ and $l \gg 0$, we get decomposition:

$$
H^{2 n+1}\left(\mathcal{K}^{e t}(\mathcal{X})[2 n-1], K_{2 n}^{e t}(\mathcal{X})\right) \cong \oplus_{i=1}^{r} H^{2 n+1}\left(\mathcal{K}^{e t}(\mathcal{X})[2 n-1], \mathbb{Z}_{l}\right)
$$


If $r=0$, so $K_{2 n}^{e t}(\mathcal{X})=0$, then the theorem obviously holds. Hence we can consider the case $r>0$. We observe that at least one of the $r$ projections of $k_{e t}^{2 n+1}(\mathcal{X}) \in H^{2 n+1}\left(\mathcal{K}^{e t}(\mathcal{X})[2 n-1], K_{2 n}^{e t}(\mathcal{X})\right)$ onto the summands of the right hand side of $(4.10)$ must have the same order as $k_{e t}^{2 n+1}(\mathcal{X})$. Take the nontorsion element $P_{i} \in K_{2 n}^{e t}(\mathcal{X})$ corresponding to this projection. By [BGK3] Th. 3.1 there are infinitely many $v$ such that $r_{v}\left(P_{i}\right)$ has order equal to $l^{k}$ which is bigger then the order of $H^{2 n+1}\left(\mathcal{K}^{e t}(\mathcal{X})[2 n-1], K_{2 n}^{e t}(\mathcal{X})\right)_{t o r}$. Observe that the groups $H^{2 n+1}\left(\mathcal{K}^{e t}(\mathcal{X})[2 n-1], K_{2 n}^{e t}(\mathcal{X})\right)$ are finitely generated $\mathbb{Z}_{l}$ modules since $K_{m}^{e t}(\mathcal{X})$ is a finitely generated $\mathbb{Z}_{l}$ module for $m>0$ and for $m=0$ we see that $H^{1}\left(\mathcal{K}^{e t}(\mathcal{X})[-1], K_{0}^{e t}(\mathcal{X})\right)=0$. Consider the following diagram

$$
\begin{gathered}
H^{2 n+1}\left(\mathcal{K}^{e t}(\mathcal{X})[2 n-1], K_{2 n}^{e t}(\mathcal{X})\right) \\
\downarrow \alpha \\
H^{2 n+1}\left(\mathcal{K}^{e t}(\mathcal{X})[2 n-1], K_{2 n}^{e t}\left(\mathcal{X}_{v}\right)\right) \\
\uparrow \beta \\
H^{2 n+1}\left(\mathcal{K}^{e t}\left(\mathcal{X}_{v}\right)[2 n-1], K_{2 n}^{e t}\left(\mathcal{X}_{v}\right)\right)
\end{gathered}
$$

Let us extract from the top vertical arrow $\alpha$ the arrow induced by the reduction of the point $P_{i}$ :

$$
H^{2 n+1}\left(\mathcal{K}^{e t}(\mathcal{X})[2 n-1], \mathbb{Z}_{l}\right) \rightarrow H^{2 n+1}\left(\mathcal{K}^{e t}(\mathcal{X})[2 n-1], \mathbb{Z} / l^{k}\right)
$$

We notice that the map (4.12) is given by reduction $\bmod l^{k}$ on coefficients. Hence the top vertical arrow of the diagram $(4.11)$ sends $k_{e t}^{2 n+1}(\mathcal{X})$ to the element of the same order.

Theorem 4.13. If Quillen-Lichtenbaum conjecture holds for $\mathcal{X}$ and $\mathcal{X}_{v}$ for all $v \notin S_{l}$ then for $n \geq 1$ and $l \gg 0$ the l-part of order of $k^{2 n+1}(\mathcal{X})$ divides the l-part of the order of $k^{2 n+1}\left(\mathcal{X}_{v}\right)$ for infinitely many $v$.

Proof. It follows by Th. 4.9 since the the reduction map

$$
K_{2 n}(\mathcal{X}) \rightarrow K_{2 n}\left(\mathcal{X}_{v}\right)
$$

is naturally compatible with the corresponding étale K-theory reduction map.

Before proceeding any further let us notice the following general lemma left as an excercise for the reader.

Lemma 4.15. Let $R$ be an infinite PID. Let $M$ be a free finitely generated $R$ module and let $\phi \in \operatorname{End}_{R}(M)$. Put $P_{\phi}(t)=\operatorname{det}\left(t \operatorname{Id}_{M}-\phi\right)$. If $M /(\phi-1) M$ is finite then the following numbers are equal:

$$
|M /(\phi-1) M|=\left|R / P_{\phi}(1)\right|
$$

where $|A|$ denotes the number of elements of a finite set $A$. 
Corollary 4.16. For an l-adic representation $\rho_{l}: G_{F} \rightarrow G L\left(T_{l}\right)$ such that $T_{l}^{F r_{v}}=$ 0 for $v \notin S_{l}$ we have equalities:

$$
\begin{gathered}
\left|H^{1}\left(g_{v}, T_{l}\right)\right|=\left|P_{v}(1)\right|_{l}^{-1} \\
\left|H^{1}\left(g_{v}, T_{l}(n)\right)\right|=\left|P_{v}\left(q_{v}^{-n}\right)\right|_{l}^{-1},
\end{gathered}
$$

where $g_{v}=G\left(\bar{k}_{v} / k_{v}\right)$.

Proof. Use Lemma 4.15 with $R=\mathbb{Z}_{l}$. To get the equality (4.17) put $M=T_{l}$ and to get the equality (4.18) put $M=T_{l}(n)$ where in both cases $\phi=F r_{v}$. The assumption $T_{l}^{F r_{v}}=0$ guaranties that the groups on the left sides of equations (4.17) and (4.18) are finite.

Corollary 4.19. For every $n \geq 1$ and $l \gg 0$ the order of $k_{e t}^{2 n+1}(\mathcal{X})$ divides the number $\left|P_{v}\left(q_{v}^{-n}\right)\right|_{l}^{-1}$ for infinitely many $v \notin S_{l}$. If the Quillen-Lichtenbaum conjecture holds for $\mathcal{X}$ and $\mathcal{X}_{v}$ for all $v \notin S_{l}$ then for every $n \geq 1$ and $l \gg 0$ the l-part of the order of $k^{2 n+1}(\mathcal{X})$ divides the number $\left|P_{v}\left(q_{v}^{-n}\right)\right|_{l}^{-1}$ for infinitely many $v \notin S_{l}$.

Proof. Consider the $l$-adic representation $\rho_{l}: G_{F} \rightarrow G L\left(T_{l}\left(J_{X}\right)\right)$. By [BGK3] Prop. 2.5 (b) and Prop. 2.6 (b) there is a natural isomorphism

$$
K_{2 n}^{e t}\left(\mathcal{X}_{v}\right) \cong H^{1}\left(g_{v} ; T_{l}\left(J_{X}\right)(n)\right)
$$

Hence by Corollary 4.16 we get equality $\left|K_{2 n}^{e t}\left(\mathcal{X}_{v}\right)\right|=\left|P_{v}\left(q_{v}^{-n}\right)\right|_{l}^{-1}$. Now the Corollary follows by Theorems 4.9 and 4.13 .

Let us now consider the case of odd K-theory. For places $v \notin S_{l}$, we consider the reduction map:

$$
r_{v}: K_{2 n+1}^{e t}(\mathcal{X}) \rightarrow K_{2 n+1}^{e t}\left(\mathcal{X}_{v}\right)
$$

Lemma 4.21. Let $n>0$ and let $l$ be an odd prime number. Assume that the group $H^{2}\left(G_{F, S_{l}} ; T_{l}\left(J_{X}\right)(n+1)\right)$ is finite. Let $k \in \mathbb{N}$ be any fixed number. Then for any nontorsion element $P \in K_{2 n+1}^{e t}(\mathcal{X})$ there are infinitely many $v$ such that $r_{v}(P)$ has order divisible by $l^{k}$.

Proof. The proof has been suggested in [BGK3] Remark 3.5. Namely we take into account Propositions 2.4, 2.5, 2.6 and exact sequences (3.7) and (3.8) of [BGK3] to observe that the claim of Lemma 4.21 about the reduction map (4.20) reduces to the corresponding claim about the reduction map:

$$
r_{v}: H^{1}\left(G_{F, S_{l}} ; \mathbb{Z}_{l}(n+1)\right) \rightarrow H^{1}\left(g_{v} ; \mathbb{Z}_{l}(n+1)\right) .
$$

But this claim about the map (4.22) has already been resolved for example in $[\mathrm{AB}]$. 
Lemma 4.23. Let $H^{2}\left(G_{F, S_{l}} ; T_{l}\left(J_{X}\right)(n+1)\right)=0$ for $n>0$. Then for $n>0$ and for $l \gg 0$ the $\mathbb{Z}_{l}$ modules $K_{2 n+1}^{e t}(\mathcal{X})$ are finitely generated, torsion free.

Proof. By Propositions 2.4, 2.6 and exact sequences (3.7) and (3.8) of [BGK3] and the assumption $H^{2}\left(G_{F, S_{l}} ; T_{l}\left(J_{X}\right)(n+1)\right)=0$, we have the following exact sequence:

$$
0 \rightarrow H^{1}\left(G_{F, S_{l}} ; \mathbb{Z}_{l}(n+1)\right) \rightarrow K_{2 n+1}^{e t}(\mathcal{X}) \rightarrow H^{1}\left(G_{F, S_{l}} ; \mathbb{Z}_{l}(n+1)\right) \rightarrow 0
$$

If we take $T_{l}:=\mathbb{Z}_{l}(n+1)$, the resulting representation is the $n+1$ twist of cyclotomic character and obviously satisfies assumptions of Lemma 4.1. Hence $H^{1}\left(G_{F, S_{l}} ; \mathbb{Z}_{l}(n+1)\right)_{l-\text { tor }}=0$ for $l \gg 0$. Hence for $l \gg 0$ the $\mathbb{Z}_{l}$-module $K_{2 n+1}^{\text {et }}(\mathcal{X})$ does not have torsion by the exact sequence $(4.24)$. The $\mathbb{Z}_{l}$-module $K_{2 n+1}^{e t}(\mathcal{X})$ is finitely generated by [DF] Prop. 5.1, p. 260.

Theorem 4.25. Let $H^{2}\left(G_{F, S_{l}} ; T_{l}\left(J_{X}\right)(n+1)\right)=0$ for $n>0$. Then for $n \geq 1$ and $l \gg 0$ the order of $k_{\text {et }}^{2 n+2}(\mathcal{X})$ divides the order of $k_{\text {et }}^{2 n+2}\left(\mathcal{X}_{v}\right)$ for infinitely many $v$. If the Quillen-Lichtenbaum conjecture holds for $\mathcal{X}$ and $\mathcal{X}_{v}$ for all $v \notin S_{l}$, then for all $n \geq 1$ and $l \gg 0$ the l-part of the order of $k^{2 n+2}(\mathcal{X})$ divides the l-part of the order of $k^{2 n+2}\left(\mathcal{X}_{v}\right)$ for infinitely many $v$.

Proof. The proof is the same as the proof of Theorems 4.9 and 4.13.

Corollary 4.26. Let $H^{2}\left(G_{F, S_{l}} ; T_{l}\left(J_{X}\right)(n+1)\right)=0$ for $n \geq 1$. Then for all $n \geq 1$ and $l \gg 0$ the order of $k_{e t}^{2 n+2}(\mathcal{X})$ divides the number $\left(\left|1-q_{v}^{n+1}\right|_{l}^{-1}\right)^{2}$ for infinitely many $v$. If the Quillen-Lichtenbaum conjecture holds for $\mathcal{X}$ and $\mathcal{X}_{v}$ for all $v \notin S_{l}$, then for all $n \geq 1$ and $l \gg 0$ the l-part of the order of $k^{2 n+2}(\mathcal{X})$ divides the number $\left(\left|1-q_{v}^{n+1}\right|_{l}^{-1}\right)^{2}$ for infinitely many $v$.

Proof. By [BGK3] Prop. 2.5 (a), (c) and Prop. 2.6 (b) there is the following exact sequence:

$$
0 \rightarrow H^{1}\left(g_{v} ; \mathbb{Z}_{l}(n+1)\right) \rightarrow K_{2 n+1}^{e t}\left(\mathcal{X}_{v}\right) \rightarrow H^{1}\left(g_{v} ; \mathbb{Z}_{l}(n+1)\right) \rightarrow 0
$$

But $\left|H^{1}\left(g_{v} ; \mathbb{Z}_{l}(n+1)\right)\right|=\left|1-q_{v}^{n+1}\right|_{l}^{-1}$ by Corollary 4.16 , hence $\left|K_{2 n+1}^{e t}\left(\mathcal{X}_{v}\right)\right|=$ $\left(\left|1-q_{v}^{n+1}\right|_{l}^{-1}\right)^{2}$. Now the Corollary follows by Theorem 4.25.

Acknowledgements: The second author would like to thank Université de Lausanne for financial support during his visit in September 2005 and Max Planck Institute in Bonn for financial support during his visit in Winter 2006. The second author was partially financed by a KBN grant and by the Marie Curie Research Training Network "Arithmetic Algebraic Geometry" MRTN-CT-2003-504917. 


\section{REFERENCES}

[A] D. Arlettaz, Algebraic K-theory of rings from a topological viewpoint, Publicacions Mathemátiques 44 (2000), 3-84.

[AB] D. Arlettaz, G. Banaszak, On the non-torsion elements in the Algebraic K-theory of rings of integers, Journal reine angew. Math. 461 (1995), 63-79.

[Au] C. Ausoni, On the Hurewicz map and Postnikov invariants of $K \mathbb{Z}$, Cohomological Methods in Homotopy Theory, (Bellaterra 1998), Progress in Math. Birkhäuser, Basel 196 (2001).

[BGK1] G. Banaszak, W. Gajda, P. Krasoń, Support problem for the intermediate Jacobians of l-adic representations, Journal of Number Theory 100 no. 1 ((2003)), 133-168.

[BGK2] G. Banaszak, W. Gajda, P. Krasoń, On reduction map for étale K-theory of curves, Homology, Homotopy and Applications, Proceedings of Victor's Snaith 60th Birthday Conference 7 (3) ((2005)), 1-10.

[BGK3] G. Banaszak, W. Gajda, and P. Krasoń, On Galois cohomology of some p-adic representations and étale K-theory of curves, Contemporary Math. AMS 241, 23-44.

[Bo] A. Borel, Stable real cohomology of arithmetic groups, Ann. Sci. École Nor. Sup. 7 (4) (1974), 235-272.

[D] P. Deligne, La cojecture de Weil, I, Publ. Math. IHES, 43292 (1974), 273-307.

[DF] W. Dwyer, E. Friedlander, Algebraic and étale K-theory, Trans. Amer. Math. Soc. 292 (1985), 247-280.

[DFST] W. Dwyer, E. Friedlander, V. Snaith, R. Thomason, Algebraic K-theory eventually surjects onto Topological K-theory, Invent. Math. 66 (1982), 481-491.

[FW] E. Friedlander and C. Weibel, An Overview of Algebraic K-Theory, Proceedings of the Workshop and Symposium: Algebraic K-Theory and its Applications, H. Bass, A. Kuku, C. Pedrini editors World Scientific, Singapore, New Jersey (1999), 1--119.

$[\mathrm{H}] \quad$ J. Huebschmann, The homotopy type of $F \Psi^{q}$. The complex and symplectic cases, Applications of Algebraic K-theory to Algebraic Geometry and Number Theory, Contemp. Math. 55, Part II (1986), 487-518.

[J] U. Jannsen, On the l-adic cohomology of varieties over number fields and its Galois cohomology., Mathematical Science Research Institute Publications, Springer-Verlag 16 (1989), 315-360.

[Q1] D. Quillen, Algebraic K- theory I, Lecture Notes in Mathematics 341 (1973), Springer Verlag.

[Q2] D. Quillen, Finite generation of the groups $K_{i}$ of rings of integers, Lecture Notes in Mathematics 341 (1973), Springer Verlag, 179-214.

[Q3] D. Quillen, On the cohomology and K-theory of the general linear groups over a finite field, Ann. of Math. 96 (2) (1972), 552-586.

[Se] J.P. Serre, Abelian l-adic representations and elliptic curve, McGill University Lecture Notes, W.A. Benjamin, New York, Amsterdam (1968).

[ST] J.P. Serre, J. Tate, Good reduction of abelian varieties and applications, Ann. of Math. 88, no 3. (1968), 492-517.

[So1] C. Soulé, K-théorie des anneaux d'entiers de corps de nombres et cohomologie étale, Inv. Math. 55 (1979), 251-295.

[So2] C. Soulé, Groupes de Chow et K-théorie de varietés sur un courps fini, Math. Ann. 268 (1984), 317-345.

[We] C. Weibel, Introduction to algebraic K-theory, book in progress at Charles Weibel home page.

[W] G.W. Whitehead, Elements of Homotopy Theory, vol. 61, Graduate Texts in Math. Springer Verlag, 1978. 
Université De Lausanne, 1015 - Lausanne, Switzerland

E-mail address: Dominique.Arlettaz@unil.ch

Department of Mathematics, Adam Mickiewicz University, Poznań, Poland

E-mail address: banaszak@amu.edu.pl 